\title{
A New Quantitative Anomalous X-ray Scattering Method for the Structural Analysis of Amorphous Thin Films
}

\author{
By Eiichiro Matsubara*, Yoshio Waseda*, Masao Mitera** \\ and Tsuyoshi Masumoto***
}

\begin{abstract}
A new quantitative method by the anomalous X-ray scattering (AXS) has been proposed for determining the local chemical environment around a specific atom in a thin multi-component film grown on a substrate. This new method using the AXS is to reduce the difficulty in the subtraction process of a substrate intensity for estimating the net intensity of a thin film, which becomes a main cause of errors of the resultant data in the conventional method. Thus, the principle for the method is fully described including the new procedure for the conversion of measured intensity data to the absolute intensity. This method has been applied to an oxide thin film $(0.3 \mu \mathrm{m}$ thick) of the $\mathrm{Bi}-\mathrm{Fe}-\mathrm{Ca}-\mathrm{O}$ system and the environmental radial distribution function around $\mathrm{Bi}$ was determined, from which the information on the valence of $\mathrm{Bi}$ was obtained.
\end{abstract}

(Received May 23, 1988)

Keywords: anomalous $X$-ray scattering, thin film, amorphous ferrite, synchrotron radiation, Seehmann-Bohlin geometry

\section{Introduction}

In the quantitative structural analysis of a thin film grown on a substrate, the scattering intensity from the substrate should be subtracted from the total scattering intensity $\left(I_{\mathrm{t}}\right)$ of a sample. The intensity of the substrate is usually estimated by applying the absorption correction $\left(A_{\mathrm{o}}\right)$ by the film to the intensity $\left(I_{\mathrm{s}}\right)$ measured in the same material as the substrate with the identical condition. Namely, the intensity from the film $\left(I_{\mathrm{f}}\right)$ is given by:

$$
I_{\mathrm{f}}=I_{\mathrm{t}}-A_{\mathrm{o}} I_{\mathrm{s}}
$$

and

$$
A_{\mathrm{o}}=\exp \left[-2 \mu_{\mathrm{f}} t_{\mathrm{f}} \frac{\sin \theta \cos (\theta-\alpha)}{\sin \alpha \sin (2 \theta-\alpha)}\right] I_{\mathrm{s}}
$$

where $\mu_{\mathrm{f}}$ and $t_{\mathrm{f}}$ are the linear absorption coefficient and thickness of the film, respec-

* Research Institute of Mineral Dressing and Metallurgy (SENKEN), Tohoku University, Sendai 980, Japan.

** Laboratory of Amorphous Magnetic Devices, Sendai 980, Japan.

*** Institute for Materials Research, Tohoku University, Sendai 980, Japan. tively. The value of $\alpha$ is the angle of incidence. Sometimes it is extremely difficult to carry out both the measurements of $I_{\mathrm{t}}$ and $I_{\mathrm{s}}$ under the exact experimental conditions especially when a single crystal is used as the substrate because of the orientational dependence of the scattering intensities. Also the constants in the absorption correction term $A_{0}$ in eq. (2) must be determined precisely. These factors frequently produce some errors for the analysis of the film in the conventional method. It is easily imagined that these errors become more serious in a thinner film of sub-micron thickness. This is a reason why the application of the X-ray diffraction technique to the structural analysis of a thin film grown on a substrate is still limited. Thus, it is desirable to develop a new method which does not include the subtraction process in eq. (1) for a more precise and successful analysis even in a thinner film. With respect to this point, the use of anomalous X-ray scattering (hereafter referred to as AXS) may result in a significant breakthrough in the quantitative structural analysis of a thin film, although the available data is still limited to only the preliminary data of an amorphous Ge film on a Si 111 wafer $^{(1)}$.

On the other hand, most of the amorphous 
materials of present interest contain more than two kinds of atoms. Therefore, the near neighbor atomic correlations of the constituents or the local chemical environments around a specific element are strongly required for describing the fine structure of multi-component systems. The main purpose of this work is to present the fundamentals of the new quantitative method for determining the local environmental structure around a specific element in a multi-component amorphous film using the AXS and its application to an oxide film of the $\mathrm{Bi}-\mathrm{Fe}-\mathrm{Ca}-\mathrm{O}$ system of $0.3 \mu \mathrm{m}$ thickness deposited on a Si 100 wafer.

\section{Principle of the New Method}

In order to determine the radial distribution function (RDF) in a non-crystalline system, the measured intensity must be converted into absolute units. In the conventional method, the so-called normalization procedure is used. Several methods for the normalization have been proposed, for example, the generalized Krogh-Moe-Norman method ${ }^{(2)}$ and the high angle method ${ }^{(3)}$. The usefulness of these methods has been recognized for the experiments on bulk samples. These normalization methods are also effectively applied to the analysis of a film grown on a substrate by subtracting the contribution of the substrate from the total intensity and obtaining the scattering intensity of the film alone, i.e. $I_{\mathrm{f}}$ in eq. (1) ${ }^{(1)}$. However, the new method using the AXS does not include this subtraction process. Although this is one of the merits of this method, the conventional normalization procedure can not be used. Thus, a new process for the conversion to the absolute intensity has been established. This new conversion procedure will first be introduced and then the method of the film analysis by applying the anomalous dispersion effect will be explained. The previous studies $^{(4)(5)}$ also strongly suggested that the most efficient use of the AXS will be done with the synchrotron radiation, because the incident $\mathrm{X}$ ray energy can be tuned in the close vicinity of the absorption edge of a desired element where the anomalous dispersion effect is very large, compared to the case of the conventional $\mathrm{X}$ - ray source. Therefore, the following discussion is also along this line.

The intensity of the incident beam is monitored by an ion chamber located in front of the sample during the course of measurements with the synchrotron radiation. The intensity of the ion chamber $\left(I_{\mathrm{m}}\right)$ is related with the power of the incident beam for a certain period of time $\left(t P_{\mathrm{o}}\right)$ by the following equation $^{(6)}$ :

$$
\begin{aligned}
t P_{\mathrm{o}}= & E_{\mathrm{p}} I_{\mathrm{m}} \exp \left(-\mu_{\mathrm{i}}\left(\frac{l_{\mathrm{t}}+l_{\mathrm{e}}}{2}\right)\right) \exp \left(-\mu_{\mathrm{a}} l_{1}\right) \\
& /\left(1-\exp \left(-\mu_{\mathrm{i}} l_{\mathrm{e}}\right)\right) / E_{\mathrm{i}} / e / G_{\mathrm{a}} / G_{\mathrm{v}}
\end{aligned}
$$

where $t$ is the counting time which is the total monitor counts in this study because of the reason explained below, $E_{\mathrm{p}}$ is the energy to form an ion-electron pair, $\mu_{\mathrm{i}}$ and $\mu_{\mathrm{a}}$ are the linear absorption coefficients of gas used for the ion chamber and air, $l_{\mathrm{t}}$ is the total length of the ion chamber and $l_{\mathrm{e}}$ is the length of the electrode in the ion chamber, $l_{1}$ is the distance from the ion chamber to the sample, $E_{\mathrm{i}}$ is the energy of photons of incidence, $e$ is the electron charge, $G_{\mathrm{a}}$ and $G_{\mathrm{v}}$ are the gain of the current amplifier and the conversion gain of the voltage-frequency converter. By using eq. (3) for $t \boldsymbol{P}_{\mathrm{o}}$, the intensity observed in the sample is given $b y^{(7)}$ :

$$
\begin{aligned}
I_{\mathrm{obs}}(Q, E)= & C P\left(\frac{\rho_{\mathrm{f}} A_{\mathrm{f}}}{M_{\mathrm{f}}} I_{\mathrm{eu}, \mathrm{f}}(Q, E)\right. \\
& \left.+\frac{\rho_{\mathrm{s}} A_{\mathrm{s}}}{M_{\mathrm{s}}} I_{\mathrm{eu}, \mathrm{s}}(Q, E)\right)
\end{aligned}
$$

where

$$
\begin{aligned}
A_{\mathrm{f}}= & \frac{\sin (2 \theta-\alpha)}{2 \mu_{\mathrm{f}} \sin \theta \cos (\theta-\alpha)} \\
& \times\left[1-\exp \left(-\frac{2 \mu_{\mathrm{f}} t_{\mathrm{f}} \sin \theta \cos (\theta-\alpha)}{\sin \alpha \sin (2 \theta-\alpha)}\right)\right] \\
A_{\mathrm{s}}= & \frac{\sin (2 \theta-\alpha)}{2 \mu_{\mathrm{s}} \sin \theta \cos (\theta-\alpha)} \\
& \times \exp \left[-\frac{2 \mu_{\mathrm{f}} t_{\mathrm{f}} \sin \theta \cos (\theta-\alpha)}{\sin \alpha \sin (2 \theta-\alpha)}\right] \\
C= & t P_{\mathrm{o}}\left(e^{4} / m^{2} / c^{4}\right)\left(w h / r^{2}\right) N_{\mathrm{A}}
\end{aligned}
$$

The quantity of $2 \theta$ is the angle between the inci- 
dent and diffracted beams, and $\mu_{\mathrm{f}}$ and $\mu_{\mathrm{s}}$ are the linear absorption coefficients of the film and substrate, respectively, $\left(e^{4} / \mathrm{m}^{2} / \mathrm{c}^{4}\right)$ $=7.9398 \times 10^{-30} \mathrm{~m}^{2}, w$ and $h$ are the width and height of the diffracted beam on the counter, $r$ is the distance from the sample to the counter, and $N_{\mathrm{A}}$ is Avogadro's constant. $P$ is the polarization term and almost equal to one for the synchrotron radiation. The values of $\rho_{\mathrm{f}}$ and $M_{\mathrm{f}}$ are the density and atomic weight of the film respectively. $\rho_{\mathrm{s}}$ and $M_{\mathrm{s}}$ are similarly defined for the substrate. $I_{\text {eu, }}(Q, E)$ and $I_{\text {eu,s }}(Q$, $E)$ are the scattering intensities from the film and substrate in absolute units, respectively.

Let us introduce the scattering factor of $\mathrm{A}$ in a n-component as expressed by:

$$
f_{\mathrm{A}}=f_{\mathrm{A}}^{\circ}+f_{\mathrm{A}}^{\prime}+i f_{\mathrm{A}}^{\prime \prime},
$$

where $f_{\mathrm{A}}^{\circ}$ corresponds to the usual atomic scattering factor of $\mathrm{A}$ at an energy sufficiently away from the absorption edge of $\mathrm{A}$, and $f_{\mathrm{A}}^{\prime}$ and $f_{\mathrm{A}}^{\prime \prime}$ are the real and imaginary parts of the anomalous dispersion terms of A. As an example, the values of $f_{\mathrm{Bi}}^{\prime}$ and $f_{\mathrm{Bi}}^{\prime \prime}$ around the $\mathrm{Bi} \mathrm{L}_{\mathrm{III}}$ absorption edge are shown in Fig. 1. The solid curves in this figure represent the theoretical values for an isolated atom ${ }^{(5)}$ computed by Cromer and Lieberman's method ${ }^{(8)}$, and the solid circles correspond to the values of $f_{\mathrm{Bi}}^{\prime}$ experimentally determined from integrated intensities of the Bragg peaks of a Bi polycrystalline plate. It is readily found that the experimental data show a good agreement with the theoretical values at the lower energy side of the absorption edge. Note that such agreement is not detected at the higher energy side of the absorption edge, mainly arising from the near edge structure and the $\mathrm{EXAFS}^{(9)}$.

Although $f_{\mathrm{Bi}}^{\prime \prime}$ is small and almost constant at the lower energy side of the edge, $f_{\mathrm{Bi}}^{\prime}$ shows the drastic change in this energy region. On the other hand, the atomic scattering factor of the substrate, which is $\mathrm{Si}$ in the present case, is almost constant at this energy region because its absorption edge is far away from the absorption edge of the A element. Also, below the absorption edge the linear absorption coefficients of $\mathrm{A}$ are almost equal. Then, the following simplification can be made as shown in the previous work $^{(1)}$.

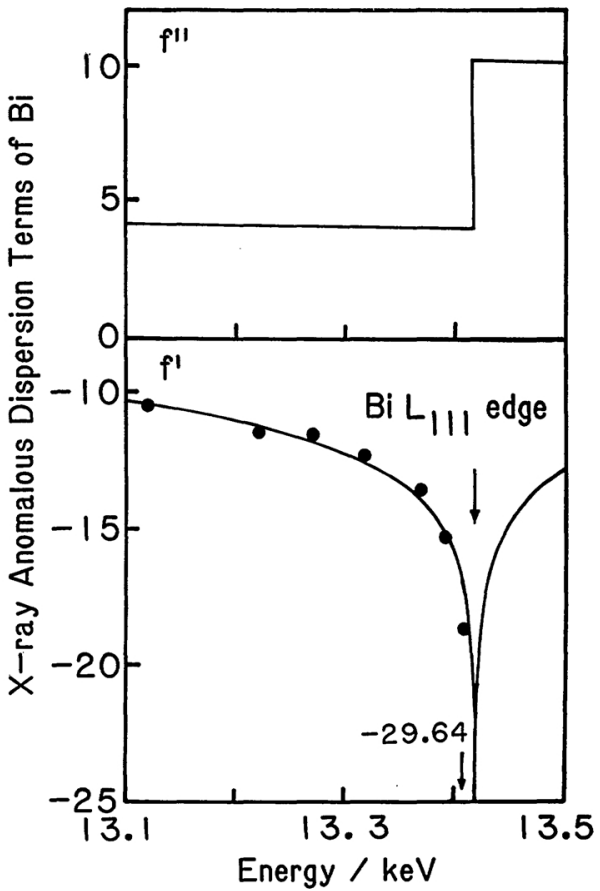

Fig. 1 Energy dependence of anomalous dispersion terms for Bi near the $\mathrm{L}_{\mathrm{III}}$ absorption edge. Solid curves correspond to the theoretical values computed by Cromer and Lieberman's method ${ }^{(8)}$.

The difference of the scattering intensities in the absolute units can be reduced to the following equation, using eq. (4).

$$
\begin{aligned}
\Delta I_{\text {eu }}(Q) & =I_{\text {eu }, \mathrm{f}}\left(Q, E_{1}\right)-I_{\text {eu }, \mathrm{f}}\left(Q, E_{2}\right) \\
& =\frac{\left(M_{\mathrm{f}} / \rho_{\mathrm{f}}\right)}{C P A_{\mathrm{f}}}\left[I_{\mathrm{obs}}\left(Q, E_{1}\right)-I_{\mathrm{obs}}\left(Q, E_{2}\right)\right]
\end{aligned}
$$

where the two energies $E_{1}$ and $E_{2}$ are below the absorption edge $\left(E_{\text {abs }}\right)$, i.e. $E_{1}<E_{2}<E_{\text {abs. }}$. By subtracting the difference of the mean square of the atomic scattering factors $\left\langle f^{2}\right\rangle$ at these two energies from the intensity difference in eq. (9), this resultant intensity function $\Delta I$ becomes:

$$
\begin{aligned}
\Delta I= & \Delta I_{\mathrm{eu}}(Q)-\left(\left\langle f^{2}\left(Q, E_{1}\right)\right\rangle-\left\langle f^{2}\left(Q, E_{2}\right)\right\rangle\right) \\
= & x_{\mathrm{A}}\left(f_{\mathrm{A}}^{\prime}\left(E_{1}\right)-f_{\mathrm{A}}^{\prime}\left(E_{2}\right)\right) \sum_{j=1}^{n} \operatorname{Re}\left[f_{j}\left(Q, E_{1}\right)\right. \\
& \left.+f_{j}\left(Q, E_{2}\right)\right] \int_{0}^{\infty} 4 \pi r^{2}\left(\rho_{\mathrm{Aj}}(r)-\rho_{\mathrm{oj}}\right) \\
& \times \frac{\sin Q r}{Q r} \mathrm{~d} r
\end{aligned}
$$


where $x_{j}$ is the atomic fraction of the $j$ th element. It is easily understood from eq. (10) that, since the scattering from the substrate and Compton scattering from the sample are eliminated by taking the difference $\Delta I$ at the two energies $E_{1}$ and $E_{2}$, the intensity $\Delta I$ enables us to directly relate the scattering intensity with the structural information of the film.

The Fourier transform of eq. (10) gives the environmental RDF around $\mathrm{A}$ of a sample film, that is,

$$
\begin{aligned}
4 \pi r^{2} \rho_{\mathrm{o}} & +\frac{2 r}{\pi x_{1}\left(f_{1}^{\prime}\left(Q, E_{1}\right)-f_{2}^{\prime}\left(Q, E_{2}\right)\right)} \int_{0}^{\infty} \frac{Q \Delta I}{W(Q)} \sin Q r \mathrm{~d} Q \\
& =4 \pi r^{2} \sum_{j=1}^{n} \frac{\operatorname{Re}\left[f_{j}\left(Q, E_{1}\right)+f_{j}\left(Q, E_{2}\right)\right]}{W(Q)} \rho_{\mathrm{Aj}}(r)
\end{aligned}
$$

where

$$
W(Q)=\sum_{j=1}^{n} x_{j} \operatorname{Re}\left[f_{j}\left(Q, E_{1}\right)+f_{j}\left(Q, E_{2}\right)\right] .
$$

Consequently, the environmental RDF around the $\mathrm{A}$ atom of the film is obtained while the difficulty in the subtraction process for the two measurements of the sample and the substrate plus sample is avoided.

\section{Experimental}

The idea for the structural analysis of amorphous thin film using AXS is likely to reduce the experimental difficulty unsolved by the conventional method and the essential equations and its relevance were now prepared. Therefore, the usefulness and validity of this new technique was tested by applying it to the structural analysis of an oxide film in the $\mathrm{Bi}-$ $\mathrm{Fe}-\mathrm{Ca}-\mathrm{O}$ system deposited on a $\mathrm{Si} 100$ single crystal. The sample preparation and the measurements of X-rays with the synchrotron radiation which are convenient for the present study are given below.

A mixture of 60 at $\% \mathrm{Fe}_{2} \mathrm{O}_{3}, 25$ at $\% \mathrm{Bi}_{2} \mathrm{O}_{3}$, and 15 at $\% \mathrm{CaO}$ was sintered at more than $1273 \mathrm{~K}$. Then it was melted at $1573 \mathrm{~K}$, quenched in water, ground and thoroughly mixed. This process was repeated two times. The total time held at $1573 \mathrm{~K}$ through the preparation was $54000 \mathrm{~s}(15 \mathrm{~h})$. Then, the powder sample which was used as a target for sputtering was pelletized in a disk of $70 \mathrm{~mm}$ diameter and 2 $\mathrm{mm}$ thickness. A single crystal silicon wafer oriented along the [001] direction cut into a rectangular shape of about $30 \mathrm{~mm}$ by $20 \mathrm{~mm}$ was used as a substrate material. The film of about
$0.3 \mu \mathrm{m}$ thickness was deposited by sputtering the target in Ar gas atmosphere at a sputtering pressure of $1021 \mathrm{~Pa}$ (10 mtorr) under a gas flow rate of $1.67 \times 10^{-7} \mathrm{~m}^{3} / \mathrm{s}$ for $7200 \mathrm{~s}(2 \mathrm{~h})$. Only an upper half of the wafer was deposited and a lower half was left as the original $\mathrm{Si}$ wafer. With this half-coated sample, the analysis by the conventional method including the subtraction process has also been carried out in order to obtain the average structure of the sample and to compare it with the environmental structure determined by the new method using AXS. The sample was mounted on a goniometer head with the vertical translation so that the intensities from the sample $\left(I_{\mathrm{t}}\right)$ and from the substrate $\left(I_{\mathrm{s}}\right)$ were measured in an exactly identical orientational relationship between the incident beam and the sample by simply changing the height of the sample. This is extremely important in order to carry out a successful structural analysis of a very thin film as the present one ${ }^{(1)}$.

The experimental set-up and its diffraction geometry are schematically described in Fig. 2 . Since a sample is irradiated with a small angle of incidence $\alpha$, the penetration depth of the

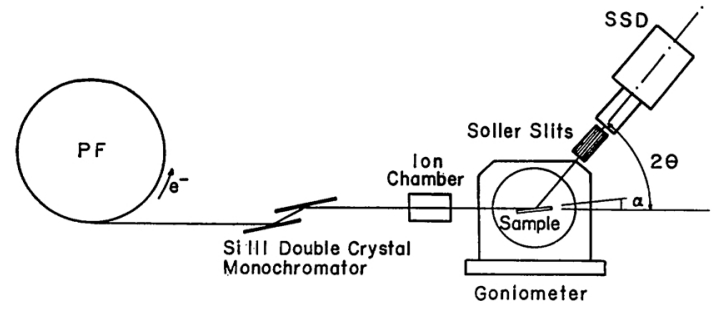

Fig. 2 Schematic diagram of experimental apparatus used for thin film measurements at the Photon Factory, Tsukuba. 
beam becomes small and scattering from a thin layer is amplified relative to scattering from the bulk. The value of $\alpha$, which was precisely determined within $0.01^{\circ}$ by aligning the sample with a parallel incident beam of a $0.02 \mathrm{~mm}$ wide, was $1^{\circ}$ in the present measurement. The product of $\mu_{\mathrm{f}} t_{\mathrm{f}}$ was experimentally determined by taking a ratio of the integrated intensities of the Bragg peak of Si 400 observed at the two different parts of the sample with and without coating. Since the thickness of the film was estimated to be $0.3 \mu \mathrm{m}$ after preparation of the sample, the density was estimated to be 5.88 $\mathrm{Mg} / \mathrm{m}^{3}$. This sample and its holder specially designed for the present measurement, and the careful determination of these constants $\alpha, \mu_{\mathrm{f}}$ and $t_{\mathrm{f}}$, made it possible to obtain the profile of the film by the subtraction process. On the other hand, in the new method the sample is fixed during the measurements. Therefore, the sample or its holder specially prepared as above is not always required.

The measurement was carried out at a beam line (7C station) in the Photon Factory of the National Laboratory for High-Energy Physics, Tsukuba Japan. The monochromatic beam was obtained using a double Si 111 crystal monochromator from which any energy of incident beam from 4 to $20 \mathrm{keV}$ was selected. Its optimum energy resolution is about $7 \mathrm{eV}$ at 10 $\mathrm{keV}$. The incident beam was monitored by a $\mathrm{N}_{2}$ gas ion chamber placed in front of the sample, and scattering intensity from the sample was measured at each angle for certain preset monitor counts in order to keep constant the total number of photons incident on the sample. A $\theta-2 \theta$ double-axis goniometer ${ }^{(10)}$ is independently driven by stepping motors with $0.0002^{\circ}$ for the $\theta$ axis and $0.0004^{\circ}$ for the $2 \theta$ axis in each step. Since the synchrotron radiation is completely polarized in the orbital plane of electrons, the goniometer was placed vertically so that the correction of the polarization effect may be ignored. Scattering intensities were measured with a Ge solid state detector so that the fluorescent radiation from the sample in the measurement near the absorption edge can be removed from the elastic scattering containing the structural information.

\section{Results and Discussion}

For comparison, the intensity measurement was at first carried out at the energy of $\mathbf{1 3 . 1 2 4}$ $\mathrm{keV}$ using the conventional method of thin film analysis for obtaining the ordinary RDF of the film. At this energy the effect of the anomalous dispersion terms on the total scattering is small and the ambiguity between the calculated and experimentally determined anomalous dispersion terms is negligible as seen in Fig. 1. The intensity profiles of both the sample and absorption-corrected substrate are shown in Fig. 3. The difference between them are also shown in this figure. This difference was corrected for the absorption by the film and converted to absolute intensity with the generalized Krogh-Moe-Norman method, followed by the correction of the Compton scattering intensity theoretically estimated ${ }^{(11)}$. The total structure factor $S(Q)$ is estimated from this resultant intensity in absolute units $I_{\mathrm{eu}}(Q)$ by the following equation:

$$
S(Q)=\left(I_{\text {eu }}(Q)-\left\langle f^{2}\right\rangle+\langle f\rangle^{2}\right) /\langle f\rangle^{2}
$$

where $\left\langle f^{2}\right\rangle-\langle f\rangle^{2}$ is the so-called Laue monotonic scattering. The Fourier transformation of $S(Q)$ gives the ordinary RDF, i.e.

$$
\begin{array}{r}
4 \pi r^{2} \rho(r)=4 \pi r^{2} \rho_{\circ}+\frac{2 r}{\pi} \int_{0}^{\infty} Q[S(Q)-1] \\
\quad \sin Q r \mathrm{~d} Q
\end{array}
$$

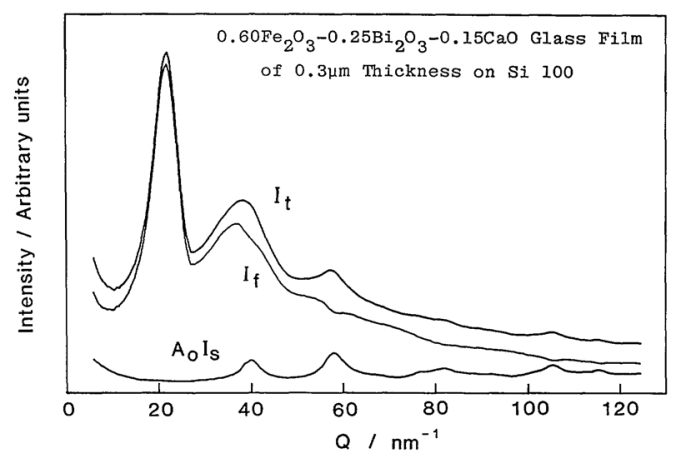

Fig. $3 \mathrm{X}$-ray scattering intensities at $13.124 \mathrm{keV}$ of $0.6 \mathrm{Fe}_{2} \mathrm{O}_{3}-0.25 \mathrm{Bi}_{2} \mathrm{O}_{3}-0.15 \mathrm{CaO}$ glass, $I_{\mathrm{t}}$, of a $\mathrm{Si}$ substrate corrected for the absorption by the glass film, $A_{\mathrm{o}} I_{\mathrm{s}}$, and the difference between them, $I_{\mathrm{f}}$. 
where $\rho(r)$ is the atomic distribution function, and $\rho_{\mathrm{o}}$ is the average number density.

Two energies 13.399 and $13.124 \mathrm{keV}$ which are respectively 25 and $300 \mathrm{eV}$ below the $\mathrm{Bi} \mathrm{L}_{\text {III }}$ absorption edge were selected for the anomalous X-ray scattering measurement. At least 200000 counts are collected at each scattering angle in both measurements. Both intensities were corrected for the escape peak intensity of the Ge solid state detector. Although the incident photon energy is below the absorption edge, the $\mathrm{Bi} \mathrm{L} \alpha$ fluorescent radiation was observed in the measurement at $13.399 \mathrm{keV}$, which mainly results from the tail of the band pass. In order to obtain a sufficiently reliable solution, such a fluorescent component should be eliminated from the total intensity ${ }^{(12)}$. The energy resolution of the solid state detector is good enough to clearly separate the L $\alpha$ component $(10.831 \mathrm{keV})$ from the elastic scattering, but insufficient to separate the $\mathrm{L} \beta$ component $(13.013 \mathrm{keV})$. However, since the intensity ratio $\mathrm{L} \beta / \mathrm{L} \alpha$ is $\mathrm{known}^{(9)}$, the intensity of the $\mathrm{L} \beta$ component was estimated and subtracted in the data reduction process by monitoring the $\mathrm{L} \alpha$ component. These intensities, followed by the correction of the absorption by air in a beam path, correspond to the intensity $I_{\text {obs }}(Q$, $E$ ) in eq. (4).

The two scattering intensities measured at the energies just below the $\mathrm{Bi} \mathrm{L}_{\mathrm{III}}$ absorption edge are shown in Fig. 4. These lines correspond to the profiles at 13.124 and $13.399 \mathrm{keV}$,

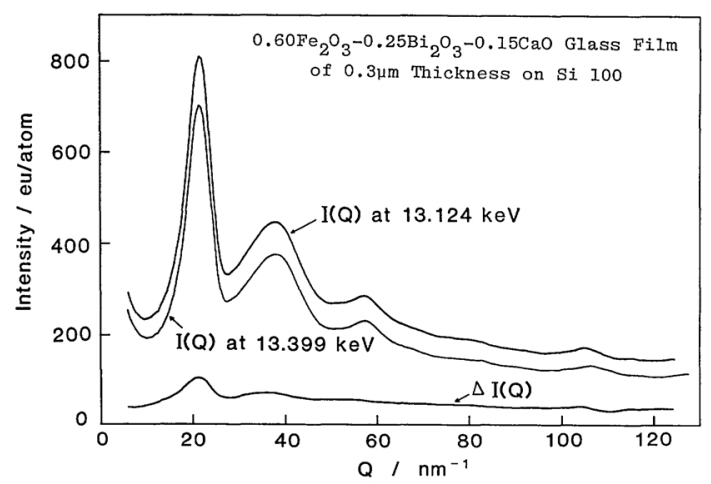

Fig. 4 Intensity profiles of $0.6 \mathrm{Fe}_{2} \mathrm{O}_{3}-0.25 \mathrm{Bi}_{2} \mathrm{O}_{3}-0.15 \mathrm{CaO}$ glass at 13.124 and $13.399 \mathrm{keV}$ which correspond to the energies of 300 and $25 \mathrm{eV}$ below the $\mathrm{Bi}_{\mathrm{III}}$ absorption edge, and a profile of the difference between them. respectively. The difference between these two profiles is surprisingly large although the thickness of the film is only $0.3 \mu \mathrm{m}$. Since only the lower energy side of the absorption edge is used in this anomalous X-ray scattering measurement, the difference between the two intensity profiles is attributed mainly to the difference of the real part of the anomalous dispersion term of $\mathrm{Bi}$. The difference of the values of $f^{\prime}$ at the $\mathrm{Bi} \mathrm{L}_{\mathrm{III}}$ absorption edge appears to be four times larger than the values expected at a K-absorption edge. Thus, the unexpectedly large difference between these two intensity profiles is understood by the large anomalous dispersion effect at the $\mathrm{L}_{\mathrm{III}}$ absorption edge. This variation corresponds to the local chemical environment around a $\mathrm{Bi}$ atom in an amorphous $\mathrm{Bi}-\mathrm{Fe}-\mathrm{Ca}-\mathrm{O}$ film.

The quantity $\Delta I$ in eq. (10) was calculated from the difference between these two intensity profiles and the result is shown in Fig. 5. Then, the environmental RDF around $\mathrm{Bi}$ was estimated from $\Delta I$ by the Fourier transformation of eq. (11). The result is shown in Fig. 6, together with the ordinary RDF calculated from the scattering measurements of Fig. 3. It is worth mentioning the following points. Since the sample of an amorphous $\mathrm{Bi}-\mathrm{Fe}-\mathrm{Ca}-$ $\mathrm{O}$ film contains four elements, there is ten possible atomic pairs; $\mathrm{Bi}-\mathrm{Bi}, \mathrm{Bi}-\mathrm{Fe}, \mathrm{Bi}-\mathrm{Ca}$, $\mathrm{Bi}-\mathrm{O}, \mathrm{Fe}-\mathrm{Fe}, \mathrm{Fe}-\mathrm{Ca}, \mathrm{Fe}-\mathrm{O}, \mathrm{Ca}-\mathrm{Ca}, \mathrm{Ca}-\mathrm{O}$ and $\mathrm{O}-\mathrm{O}$. The ordinary RDF is reflected on these ten partial structural information, while the environmental $\mathrm{RDF}$ around $\mathrm{Bi}$ obtained by the present AXS contains mainly four partials of $\mathrm{Bi}-\mathrm{Bi}, \mathrm{Bi}-\mathrm{Fe}, \mathrm{Bi}-\mathrm{Ca}$ and $\mathrm{Bi}-\mathrm{O}$ pairs. Then, the

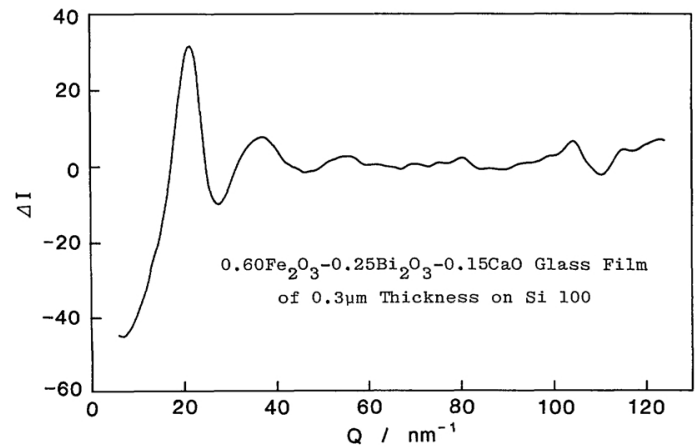

Fig. 5 Profile of the intensity difference defined in eq. (10). 


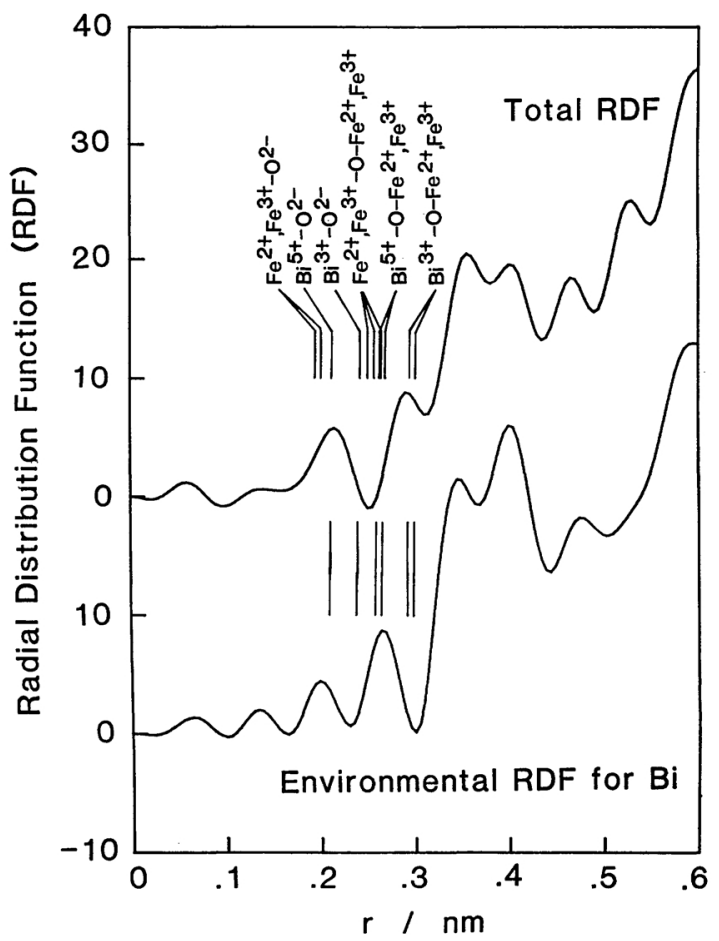

Fig. 6 Environmental radial distribution function (RDF) and ordinary $\mathrm{RDF}$ of $0.6 \mathrm{Fe}_{2} \mathrm{O}_{3}-0.25 \mathrm{Bi}_{2} \mathrm{O}_{3}-0.15 \mathrm{CaO}$ glass.

relatively easy interpretation of the result is allowed in the environmental RDF data of multicomponent systems. A discussion of the detailed structure for an amorphous thin film of the $\mathrm{Bi}-\mathrm{Fe}-\mathrm{Ca}-\mathrm{O}$ system is outside the scope of the present study. However, the following observation could be made only from the results of Fig. 6.

The first peak appears at almost the same place in both RDFs. However, the second peak in the environmental RDF shifts to a lower $r$ value. This system consists of four elements, that is, $\mathrm{Fe}, \mathrm{Bi}, \mathrm{Ca}$ and $\mathrm{O}$. Since the scattering factors of $\mathrm{Ca}$ is less than $\mathrm{Fe}$ and $\mathrm{Bi}$, and the atomic fraction of $\mathrm{Ca}$ is much less than the other three elements, the atomic pairs including $\mathrm{Ca}$ were ignored in the interpretation of the RDFs. The distances of some cation- $\mathrm{O}^{2-}$ pairs and cation-cation pairs bonded by an oxygen ion were estimated, referring ionic radii to Shannon's values ${ }^{(13)}$. The distances of some possible pairs are indicated in Fig. 6. It is found that $\left(\mathrm{Fe}^{2+}, \mathrm{Fe}^{3+}\right.$ or $\left.\mathrm{Bi}^{5+}\right)-\mathrm{O}^{2-}$ pairs are at the first peak. Pairs of $\left(\mathrm{Fe}^{2+}\right.$ or $\left.\mathrm{Fe}^{3+}\right)$ and $\left(\mathrm{Fe}^{2+}\right.$ or $\left.\mathrm{Fe}^{3+}\right)$, or $\left(\mathrm{Bi}^{3+}\right.$ or $\left.\mathrm{Bi}^{5+}\right)$ and $\left(\mathrm{Bi}^{3+}\right.$ or $\mathrm{Bi}^{5+}$ ) through $\mathrm{O}^{2-}$ are possibly included at the second peak in the total RDF. The environmental RDF determined at the $\mathrm{Bi} \mathrm{L}_{\mathrm{III}}$ absorption edge represents the atomic configurations around $\mathrm{Bi}$. For example, $\left(\mathrm{Bi}^{3+}\right.$ or $\left.\mathrm{Bi}^{5+}\right)-\mathrm{O}^{2-}$, $\left(\mathrm{Bi}^{3+}\right.$ or $\left.\mathrm{Bi}^{5+}\right)-\mathrm{O}-\left(\mathrm{Fe}^{2+}\right.$ or $\left.\mathrm{Fe}^{3+}\right)$, or $\left(\mathrm{Bi}^{3+}\right.$ or $\left.\mathrm{Bi}^{5+}\right)-\mathrm{O}-\left(\mathrm{Bi}^{3+}\right.$ or $\left.\mathrm{Bi}^{5+}\right)$ are possibly included. Thus, it is easily seen from Fig. 6 that the first and second peak are explained by pairs with $\mathrm{Bi}^{5+}$ but not by pairs with $\mathrm{Bi}^{3+}$. Consequently, in this system, $\mathrm{Bi}$ atoms become $\mathrm{Bi}^{5+}$ instead of $\mathrm{Bi}^{3+}$. Mitera et al. ${ }^{(14)}$ concluded that $\mathrm{Fe}$ atoms in this glass are a mixture of $\mathrm{Fe}^{2+}$ and $\mathrm{Fe}^{3+}$. Therefore, it is plausible that a charge transfer could happen in other cations.

For further discussion about the mechanism of magnetization in this oxide thin film, the environmental RDF around $\mathrm{Fe}$ is, of course, required in a similar manner, using the anomalous dispersion effect of $\mathrm{Fe}$ atoms near the Fe $\mathrm{K}$ absorption edge; this is under investigation. It is rather the intention of the present work to indicate that the potential capability of the quantitative method for the structural analysis of amorphous film using AXS has been demonstrated by means of an oxide thin film of sub-micron thickness grown on a substrate. The efficient use of the $\mathrm{L}$ absorption edge is also evident because of the much larger anomalous dispersion effect than the $\mathrm{K}$ absorption edge.

\section{Concluding Remarks}

A new method using anomalous X-ray scattering for the structural analysis of amorphous thin films of less than $1 \mu \mathrm{m}$ thickness has been proposed with the essential equations and the procedure for the conversion of the measured intensity data to the absolute intensity. The capability of this new method was clearly demonstrated by determining the local chemical environment around $\mathrm{Bi}$ in an oxide film of the $\mathrm{Bi}-\mathrm{Fe}-\mathrm{Ca}-\mathrm{O}$ system grown on a $\mathrm{Si}$ 100 wafer without the difficulty of the subtraction process for the two measurements of the sample and the substrate plus sample which is 
necessary for the conventional method. The present work is orientated towards amorphous thin films. However, the new method using AXS presently proposed is also applicable to crystalline systems, when the purpose is to estimate the environmental radial distribution function around a specific element, as exemplified by the results of a bulk sample of the $\mathrm{Y}-\mathrm{Ba}-\mathrm{Ca}-\mathrm{O}$ superconductor ${ }^{(15)}$. Thus, it may be interesting to extend the present method to the structural characterization of thin films in a variety of states, so that its usefulness and validity may be tested in a rather wider base.

\section{Acknowledgments}

A part of this research was supported by the Ishihara-Asada Research Foundation and Iron and Steel Institute of Japan in 1987. We (EM and YW) particularly want to thank the staff of the Photon Factory, National Laboratory for High Energy Physics, Drs. T. Ishikawa and M. Nomura and Profs. T. Matsushita, H. Iwasaki and M. Ando.

\section{REFERENCES}

(1) E. Matsubara, Y. Waseda and H. Itozaki: Trans. Japan. Inst. Metals, 29 (1988), 1.
(2) C. N. J. Wagner, H. Ocken and M. L. Joshi: Z. Naturforsch., 20a (1965), 325.

(3) N. S. Gingrich: Rev. Mod. Phys., 15 (1943), 90.

(4) P. H. Fuoss, P. Eisenberger, W. K. Warburton and A. Bienenstock: Phys. Rev. Lett., 46 (1981), 1537.

(5) Y. Waseda: Novel Application of Anomalous $X$-ray Scattering for Structural Characterization of Disordered Materials, Springer-Verlag, Heiderberg, (1984), p. 162.

(6) E. Matsubara and P. Georgopoulos: J. Appl. Cryst., 18 (1985), 377.

(7) L. H. Schwartz and J. B. Cohen: Diffraction from Materials, Academic Press, New York, (1977) p. 520.

(8) D. T. Cromer and D. Lieberman: J. Chem. Phys., 53 (1970), 1891.

(9) B. K. Teo: EXAFS Basic Principles and Data Analysis, Springer-Verlag, Berlin Heidelberg, (1986) p. 15.

(10) Y. Waseda, E. Matsubara and K. Sugiyama: Sci. Rep. Res. Inst. Tohoku Univ., 34A (1987), 1.

(11) International Tables for X-ray Crystallography, Vol. IV, The Kynoch Press, Birmingham, 1974.

(12) S. Aur, D. Kofalt, Y. Waseda, T. Egami, R. Wang, H. S. Chen and B. K. Teo: Solid State Commun., 48 (1983), 111.

(13) R. D. Shannon and C. T. Prewitt: Acta Crystallogr., B25 (1969), 925.

(14) M. Mitera, M. Mimura, S. Ohta and S. Masuda: Final Report of Sozo-Kagaku-Gijutsu-Suishin-Jigyo (Masumoto Project), (1986), p. 7.

(15) K. Sugiyama, E. Matsubara, Y. Waseda, A. Inoue and T. Masumoto: J. Mat. Sci. Lett., 7 (1988), 450. 